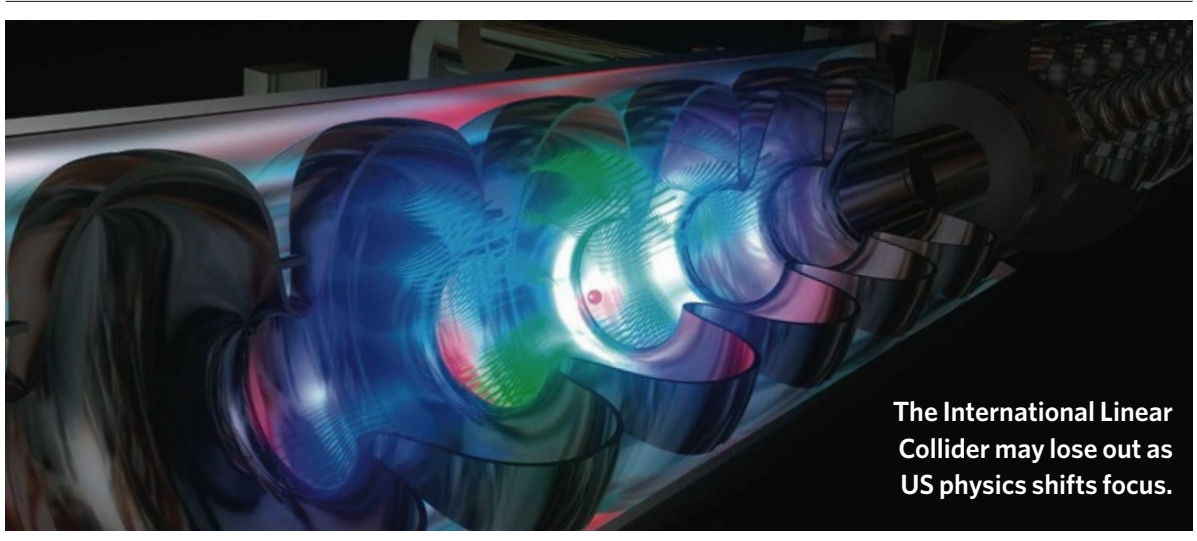

\title{
Physicists to target neutrinos
}

The new focus for America's high-energy physics should be an elusive one: the zippy, chargeless, near-massless neutrino, according to a report that provides the US Department of Energy (DOE) and the National Science Foundation (NSF) with a roadmap for the next decade.

The report, written by the Particle Physics Project Prioritization Panel (P5) and endorsed on 29 May by a DOE and NSF advisory committee, suggests that the US physics programme should concentrate on the fertile terrain of neutrino physics rather than focusing all its efforts on the highenergy frontier explored by colliders.

The report also seems to spell the end for US ambitions to host the International Linear Collider (ILC), a planned smasher of electrons and positrons that would complement the Large Hadron Collider (LHC), which is to begin operations this summer at CERN, the European particle-physics laboratory near Geneva. Some physicists wanted to pursue an aggressive schedule for the ILC. But until the LHC starts producing results, it will be unclear whether the ILC has been designed to be powerful enough.

Yale University's Charles Baltay, who chaired the $\mathrm{P} 5$ panel, says that research and development should continue for the ILC, but that it was no longer "front and centre". "This was obviously the thorniest issue the panel faced," says Baltay. "We spent four months arguing about this."

Instead, the $\mathrm{P} 5$ report recommends more work on the neutrino, which was discovered to have mass only a decade ago. Physicists want to understand how the three known neutrino 'flavours' morph from one type to another. Some physicists hope that by understanding neutrinos and their

antiparticles, they will be able to explain why the Universe ended up being made of matter rather than antimatter.

To this end, the $\mathrm{P} 5$ report recommends starting construction of $\mathrm{NO} v \mathrm{~A}$, a programme that would send a neutrino beam from Fermilab in Batavia, Illinois, to detectors in an underground mine in Minnesota. The report endorses also building a more powerful neutrino beam that would travel farther, from Fermilab to the planned Deep Underground Science and Engineering Lab (DUSEL) in the Homestake Mine of South Dakota. The farther the neutrinos travel, the more sensitive the experiments are.

But the future of the ILC, at least for it being hosted by the United States, looks $\mathrm{dim}$. The recommendations to continue ILC research and development are "okay", says Barry Barish, a high-energy physicist at the California Institute of Technology in

"We spent four months arguing about this."
Pasadena, who heads the ILC design effort. "But it isn't an aggressive programme to bring the ILC to the United States.” Just last year Barish spoke of starting ILC construction by 2012; now he just wants to have a construction proposal by 2012 . Japan is now the front runner for hosting the project, Barish says, with CERN also being a contender after it finishes planned upgrades to the LHC.

Given the sharp budget cuts for highenergy physics in 2008, there is no guarantee that the US Congress will pay any attention to the physicists' roadmap, which considers four budget scenarios. "What happens inside Washington happens," says Melvyn Shochet, a physicist at the University of Chicago, Illinois, and chair of the DOE and NSF advisory committee. "But you need to be able to make the case."

Eric Hand
ON THE RECORD

\section{cclearly, having a working toilet is a priority for us.)}

Shuttle payload manager Scott Higginbotham, on the failure of the toilet system on the International Space Station. The three astronauts aboard used the one on the moored Soyuz capsule instead.

\section{SCORECARD}

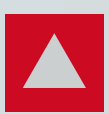

Skydiving balloon A $\$ 200,000$ helium balloon, supposed

to take French daredevil Michel Fournier $\mathbf{4 0}$ kilometres up for a record-setting sky-dive, launched successfully last week - but without Fournier. A technical problem led to its premature release. It was Fournier's third failed attempt at the jump, but he says that he'll try again in August.

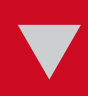

Party balloons

The California Senate has voted to ban

helium-filled metallic balloons because they, too, frequently escape, and can get tangled in electrical lines. They have apparently caused hundreds of power outages in recent years.

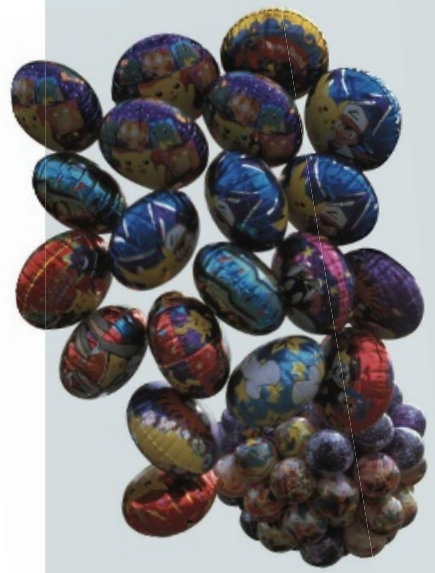

NUMBER CRUNCH

$\mathbf{8 3 0}$ years ago the Tower of Pisa first began to lean. It has now officially stopped moving.

28 years ago, the latest attempts to un-tilt the tower began.

70 tonnes of earth were shifted to prop up it up.

300 years is how long it is expected to remain stable at its new lean, which is about the same tilt it had in 1700 . 